\title{
Mixed connective tissue disease presenting as trigeminal neuropathy
}

\author{
W. M. EDMONDSTONE \\ M.B., M.R.C.P. \\ D. K. PRICE \\ M.B. B.S.
}

\author{
T. H. SHEPHERD \\ M.B. B.S. \\ W. W. GoODDY \\ M.D., F.R.C.P.
}

Royal Naval Hospital, Haslar, Gosport, Hants

\begin{abstract}
Summary
A 25-year-old man presented with an isolated trigeminal neuropathy 13 months before developing myalgia and lymphadenopathy. The onset of Raynaud's phenomenon 2 months later suggested a diagnosis of mixed connective tissue disease (MCTD) and this was confirmed by high serum titres of speckled pattern antinuclear antibody, and antibody to ribonuclear protein.
\end{abstract}

\section{Introduction}

Neurological manifestations occur rarely in mixed connective tissue disease (MCTD) a condition which was first described by Sharp, Irvin and Tan in 1972. The hallmarks of this condition, which shares overlapping features of systemic lupus erythematosus (SLE), systemic sclerosis, and polymyositis, are Raynaud's phenomenon, arthralgia and lymphadenopathy. Sclerodactyly, myositis, swelling of the hands and swallowing defects are found commonly, and the disease is characterized by the presence in the serum of an antibody specific for the ribonuclear protein (RNP) component of extractable nuclear antigen (ENA). Renal involvement is rare, and MCTD is considered to be a benign disorder with a good response to treatment and a favourable prognosis.

Although there are several reports of trigeminal neuropathy occurring as an early manifestation of MCTD, other features of the syndrome have been present at the time of diagnosis. A case is now reported in which an isolated trigeminal sensory neuropathy preceded the rest of the syndrome by at least one year.

\section{Case report}

A 25-year-old radio operator in the Royal Navy developed a sensation of numbness and tingling affecting the right eye and cheek in March 1979. The numbness extended to the side of the nose and upper lip, and he described the sensation as being similar to the effects of a dental anaesthetic. Examination revealed an absent right cornea! reflex, with reduction of sensory perception in the area supplied by the first and second divisions of the right fifth cranial nerve. The jaw jerk, motor component of the fifth nerve and remainder of the nervous system were normal. General examination was also normal.

Full blood count and ESR, glucose, urea and electrolytes, liver function tests, protein electrophoresis and syphilitic serology were all normal. CSF was normal as were $\mathrm{X}$-rays of chest, skull and sinuses. Enhanced CT scan showed minimal enlargement of the right ventricle, and an air encephalogram showed a normal posterior fossa.

In April 1980 he developed a febrile illness with generalized myalgia and widespread lymphadenopathy. His full blood count and ESR were normal, and Monospot, virology titres and Toxoplasma dye tests were negative. Two months later he developed Raynaud's phenomenon and was found to have shiny skin over the fingers. The lymphadenopathy persisted. Antinuclear antibody was present in a titre of $1: 640$ with a speckled pattern, and antibody in his serum to RNP was strongly positive. The C3 component of complement was slightly reduced at $6.4 \mathrm{~g} / 1$ (normal range 8.1-15.0 g/l) but antibodies to double stranded DNA were negative.

He was treated with prednisolone $20 \mathrm{mg}$ daily and within 3 months his corneal reflex had returned, with some improvement in sensation in his face. Although the lymphadenopathy resolved, there was no improvement in the Raynaud's phenomenon.

\section{Discussion}

Isolated trigeminal neuropathy may occur as a result of trauma, tumours, demyelinating diseases or connective tissue disorders. It may also be idiopathic. Ashworth and Tait (1971) reported six patients with facial numbness of whom three had systemic sclerosis, two had SLE with features of 
systemic sclerosis, and one had polymyositis. It is possible that two of these patients had what would now be called MCTD.

There have been several reports of trigeminal neuropathy occurring in MCTD and three cases were found in one series of twenty patients (Bennett and Spargo 1977). Vincent and Van Houzen (1980) described a case in which trigeminal sensory neuropathy and bilateral carpal tunnel syndrome heralded the onset of MCTD, but this patient also had swollen hands with tight skin. Searles, Mladirich and Messner (1978) reported trigeminal neuropathy developing 2 weeks after the onset of Raynaud's phenomenon in a young woman in whom MCTD was later diagnosed. Similarly, the concurrent development of trigeminal neuropathy and Raynaud's phenomenon at the onset of MCTD was also reported by Lagarde and Lamotte-Barrillon (1979). The present patient, however, developed isolated trigeminal neuropathy in the absence of any other features of MCTD. There was a delay of at least 12 months before the development of lymphadenopathy followed by Raynaud's phenomenon, pointed to the correct diagnosis.

Neurological complications occur commonly in connective tissue disorders, and the peripheral nerves may be involved in a sensory or mixed sensorimotor neuropathy, or in a mononeuritis multiplex. The cause of the neuropathy in MCTD is not known, nor is it known why the disease has a predilection for the trigeminal nerve. Although there have been no post-mortem reports to confirm this, a vasculitis of the trigeminal nerve may cause the neuropathy in MCTD. Weiss et al. (1978) reported a case of fatal transverse myelitis in MCTD in which post-mortem 2 demonstrated multifocal necrosis of the cord due to $\mathbb{\otimes}$ vasculitis.

There is a long list of causes of isolated trigeminal neuropathy (Editorial, 1969). The present authors suggest that MCTD should be considered in caseso where the diagnosis remains unclear.

\section{References}

AshWORTH, B. \& TAIT, G.B.W. (1971) Trigeminal neuro- ొొ pathy in connective tissue disease. Neurology (Minne- $-\infty$ apolis), 21, 609.

BENNETT, R.M. \& SPARgo, B.H. (1977) Neuropyschiatric problems in mixed connective tissue disease. In: Pro- $\vec{\omega}$ ceedings of the XIV International Congress of Rheumatology Bethesda, International Congress of Rheumatology, p. 37.

EDITORIAL (1969) Isolated trigeminal neuropathy. Lancet, $\frac{0}{3}$ ii, 1347.

LAGARDE, P. \& LAMOTTE-BARRILlon, S. (1979) Isolated trigeminal neuropathy revealing the presence of Sharp's syndrome or MCTD (mixed connective tissue disease). Annales de Médicine interne, 130, 337.

Searles, R.P., Mladirich, E.K. \& Messner, R.P. (1978) Isolated trigeminal sensory neuropathy: early manifestion mixed connective tissue disease. Neurology (Minneapolis), 음 28, 1286.

SharP, G.C., IRvin, W.S. \& TAN, E.M. (1972) Mixed connective tissue disease-an apparent distinct rheumatic 0 disease syndrome associated with a specific antibody to an extractable nuclear antigen (ENA). American Journal of $\vec{\emptyset}$
Medicine, 52, 148 .

Vincent, F.M. \& VAN Houzen, R.N. (1980) Trigemi sensory neuropathy and bilateral carpal tunnel syndrome: the"initial manifestation of mixed connective tissue diseas. Journal of Neurology, Neurosurgery and Psychiatry, 4 458.

Weiss, T.D., Nelson, J.S., Woolsey, R.M., ZuCKNer, J. \& BALDASARRE, A.R. (1978) Transverse myelitis in mixed $\mathbb{D}$ connective tissue disease. Arthritis and Rheumatism, 21, 982. 